\title{
Restored expression of the atypical heat shock protein H11/HspB8 inhibits the growth of genetically diverse melanoma tumors through activation of novel TAK1-dependent death pathways
}

\author{
CC Smith ${ }^{1,3}$, KS Lee ${ }^{1,3}$, B Li ${ }^{1}$, JM Laing ${ }^{1}$, J HersI ${ }^{1}$, M Shvartsbeyn ${ }^{1,2}$ and L Aurelian ${ }^{\star, 1}$
}

\begin{abstract}
Melanoma is an aggressive and drug-resistant cancer in need of improved therapeutic strategies. Restored expression of transcriptionally silenced genes is a potential approach, but it is limited by the genetic diversity of the melanoma tumors. The atypical heat shock protein $\mathrm{H} 11 / \mathrm{HspB} 8$ has kinase activity and is silenced in melanoma through aberrant DNA methylation. We report that its restored expression induces the death of genetically diverse melanoma lines and inhibits tumor growth through the activation of novel TAK1-dependent death pathways. These include (i) caspase-1 activation independent of the inflammasome through upregulation of apoptosis-associated speck-like protein containing a CARD (ASC), (ii) Beclin-1 upregulation through phosphorylation of mammalian target of rapamycin (mTOR) at S2481 and (iii) apoptosis caused by caspase-1-mediated Beclin-1 cleavage. These data extend current understanding of cell death-associated functions, underscore the strong therapeutic promise of H11/HspB8 and identify TAK1 as a potential intervention target in melanoma.
\end{abstract}

Cell Death and Disease (2012) 3, e371; doi:10.1038/cddis.2012.108; published online 16 August 2012

Subject Category: Cancer

Melanoma is a commonly diagnosed highly aggressive and drug-resistant cancer that accounts for approximately $79 \%$ of cancer skin deaths worldwide. ${ }^{1}$ Restored expression of genes epigenetically silenced during melanoma development is a therapeutic option, but a major complication is the established genetic diversity of the melanoma tumors. Global genome analyses identified a large number of hypermethylated genes, but they varied widely among distinct melanoma lines and their ability to induce cell death is largely unknown. ${ }^{2}$

Our studies focus on the small heat shock protein (Hsp) $\mathrm{H} 11$ (Entrez ID \# 26353, also known as Hsp22 and HspB8), which was originally cloned in our laboratory. $\mathrm{H} 11 / \mathrm{HspB} 8$ differs from canonical family members in biological and functional properties that include intrinsic kinase activity, association with the plasma membrane, failure to translocate to the nucleus upon heat shock and the presence of nuclear export sequences. It induces the proliferation of keratinocytes and cardiomyocytes but causes cell cycle arrest in melanocytes. ${ }^{3-6} \mathrm{H} 11 / \mathrm{HspB} 8$ is silenced in a high proportion of human melanomas, 6,7 in human prostate cancer and Ewing's sarcoma cells ${ }^{7}$ and in hematological malignancies ${ }^{8}$ through aberrant DNA methylation, and its restored expression was associated with caspase-3 activation and apoptosis. ${ }^{6,7,9} \mathrm{We}$ report that restored expression of $\mathrm{H} 11 / \mathrm{HspB} 8$ induces the death of genetically diverse melanoma cells through the activation of line-specific and novel death pathways, which initiate with the activation of the MAP3K family member TGFbeta-activated kinase 1 (TAK1), the association of which with

\footnotetext{
${ }^{1}$ Department of Pharmacology, University of Maryland, School of Medicine, 655 West Baltimore Street, Baltimore, MD, USA

*Corresponding author: L Aurelian, Department of Pharmacology, University of Maryland, School of Medicine, 655 West Baltimore Street, Baltimore, MD 21201-1559, USA. Tel: 410706 3895; Fax: 410706 2513; E-mail: laurelia@umaryland.edu

${ }^{2}$ Current address: Department of Pathology, New York University, 522 First Avenue, Smilow, 301C, New York, NY 10016, USA.

${ }^{3}$ These authors contributed equally to this work.

Keywords: caspase-1; caspase-3/7; apoptosis; ASC; Beclin-1; mTOR

Abbreviations: TAK1, transforming growth factor beta-activated kinase 1; pMKK6, phosphorylated mitogen-activated protein kinase 6; ASC, apoptosis-associated speck-like protein containing a CARD; CARD, C-terminal caspase-recruitment domain; Hsp, heat shock protein; MAP3K, mitogen-activated 3 protein kinase; TGF $\beta$, transforming growth factor beta; Dox, doxycyline; TUNEL, terminal deoxynucleotidyl transferase dUTP nick end labeling; AKT, also known as protein kinase B; s.C., subcutaneous; PK, protein kinase; TNF- $\alpha$, tumor necrosis factor $\alpha$; TRAF6, tumor necrosis factor-receptor-associated factor 6; TAB1, TAK1 binding protein 1; TAB2, TAK1 binding protein 2; ZVAD-fmk, carbobenzoxy-valyl-alanyl-aspartyl-[O-methyl]-fluoromethylketone carbobenzoxy-valyl-alanyl-aspartyl-[O-methyl]-fluoromethylketone; Z-DQMD-fmk, benzyloxycarbonyl-Asp(OMe)-Gln-Met-Asp(OMe)-fluoromethylketone; IL-1 $\beta$, interleukin 1 beta; IL-18, interleukin 18; YVAD-CHO, Ala-Ala-ValAla-Leu-Leu-Pro-Ala-Val-Leu-Leu-Ala-Leu-Leu-Ala-Pro-Tyr-Val-Ala-Asp aldehyde peptide; JNK, c-jun N terminal-kinase; aODN, antisense phosphothiorate oligonucleotide; sODN, sense phosphothiorate oligonucleotide; mTOR, mammalian target of rapamycin; mTORC1, mammalian target of rapamycin complex 1; pmTORS2481, mTOR phosphorylated at serine 2481; pmTORS2448, mTOR phosphorylated at serine 2488; COXIV, cytochrome coxidase IV; p38MAPK, p38 mitogenactivated protein kinase; DMEM, Dulbecco's modified Eagle's medium; FBS, fetal bovine serum; ERK 1, extracellular signal regulated kinase 1; ERK2, extracellular signal regulated kinase 2; JIP, c-jun N terminal-kinase interacting protein; HEK293, human embryonic kidney cells; GAPDH, glyceraldehyde 3-phosphate dehydrogenase; TMR-red-labeled dUTP, tetramethylrhodamine red-labeled dUTP; DAPI, 4',6-diamidino-2-phenylindole; RAP1, Ras-related protein 1; ELISA, enzymelinked immunosorbent assay; NHM, normal adult human melanocytes; IgG, immunoglobulin G
}

Received 03.5.12; revised 28.6.12; accepted 02.7.12; Edited by A Stephanou 
cancer suppression is still poorly understood. ${ }^{10-12}$ The novel pathways include (i) ASC-mediated caspase-1 activation independent of the inflammasome, (ii) Beclin-1 upregulation through mTOR phosphorylation at S2481 and (iii) apoptosis caused by caspase-1-mediated Beclin-1 cleavage. These data extend current understanding of death-associated functions and underscore the promise of $\mathrm{H} 11 / \mathrm{HspB} 8$ as an epigenetics-based melanoma therapeutic.

\section{Results}

Targeted H11/HspB8-restored expression inhibits the growth of A375 and A2058 xenografts. We studied melanoma cells A375 and A2058 that have distinct genetic signatures (Supplementary Figures S1 and S2). The cells were stably transfected with tet-inducible $\mathrm{H} 11 / \mathrm{HspB} 8$, an established targeted gene expression strategy to induce conditional cell death, which is more clinically relevant than ectopic delivery (Supplementary Materials and methods). Dox treatment of untransfected A375 and A2058 cells (2, 5 or $10 \mu \mathrm{g} / \mathrm{ml}$; 3 days) did not reduce the number of melanoma $(\mathrm{S} 100+)$ cells, induce apoptosis (TUNEL) or restore endogenous $\mathrm{H} 11 / \mathrm{HspB} 8$ expression (Figure 1a), and it did not activate the Akt or MAPK pathways (Supplementary Figure S1A). This is consistent with previous reports that Dox does not cause melanoma cell death at the dose used in our studies $(5 \mu \mathrm{g} / \mathrm{ml}),{ }^{13}$ but death was induced by ectopic delivery of $\mathrm{H} 11 / \mathrm{HspB8} .^{7}$ Significantly, the growth of a

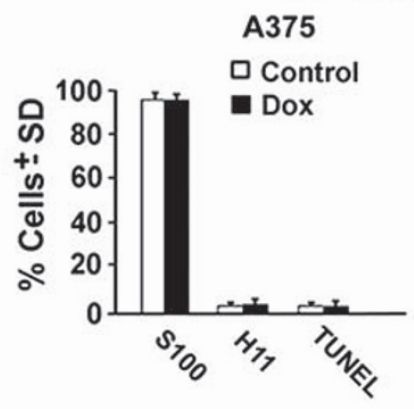

b

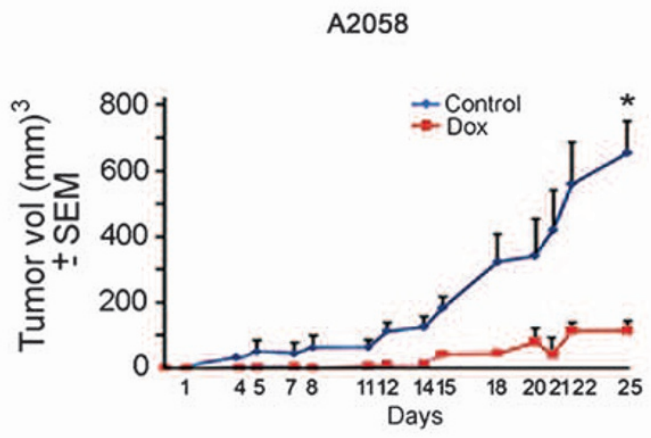

C

A2058

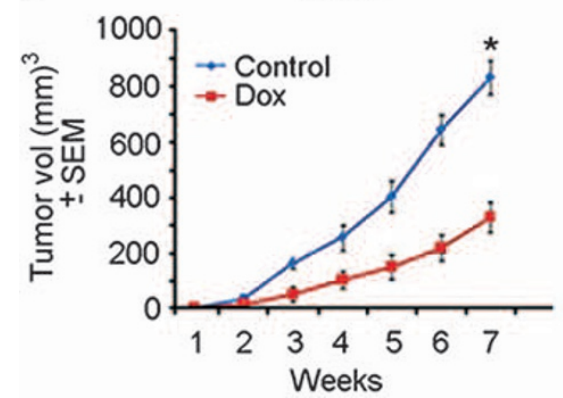

Untransfected Cell Lines

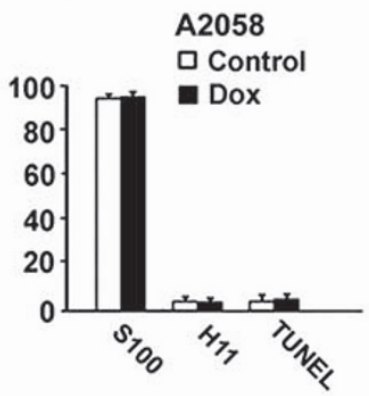

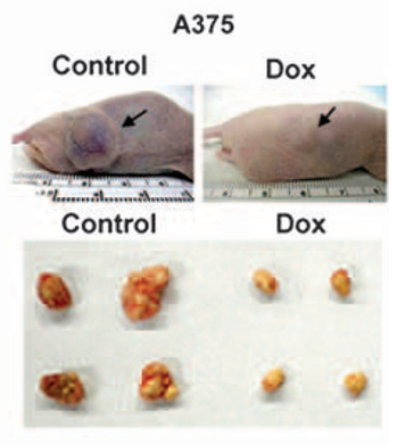

A375

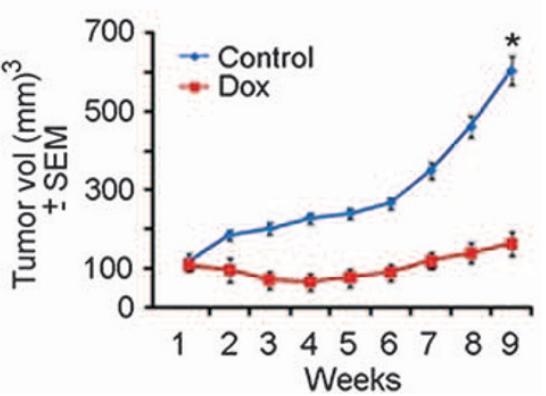

Figure 1 Restored H11/HspB8 expression inhibits the growth of A375 and A2058 xenografts. (a) Untransfected A375 and A2058 cells were treated with Dox (5 $\mu \mathrm{g} / \mathrm{ml}$; 3 days) and stained in triple immunofluorescence with antibodies to S100 (AlexaFluor350-conjugated secondary antibody; blue) and H11/HspB8 (AlexaFluor 488-conjugated secondary antibody; green) and TUNEL (TMR-red labeled dUTP). The cells were counted in five randomly selected $3 \mathrm{~mm}^{2}$ fields ( 250 cells each) and the \% positive cells calculated relative to the total number of melanoma cells $(\mathrm{S} 100+)$. Results are expressed as \% positive cells \pm S.D. Similar results were obtained for Dox at 2 or $10 \mu \mathrm{g} / \mathrm{ml}$. (b) Balb/c (nu/nu) mice given A2058 or A375 cells stably transfected with tet-regulated H11/HspB8 (5 $\times 10^{6}$ cells) by subcutaneous injection into both flanks were randomly divided into two groups ( $n=10$ tumors/group) and left untreated or given Dox $(2 \mathrm{mg} / \mathrm{ml})$ in the drinking water. Tumor volume calculated as described in the Materials and methods section is shown for A2058 xenografts. Similar results were obtained for A375 xenografts ( $\left.{ }^{\star} P<0.001\right)$. Mice bearing A375 xenografts that were untreated (control) or treated with Dox (Dox) were photographed on day 25 and representative excised tumors are shown. (c) Mice given $5 \times 10^{5}$ stably transfected cells were similarly followed and tumor volume calculated $\left({ }^{\star} P<0.001\right)$ 

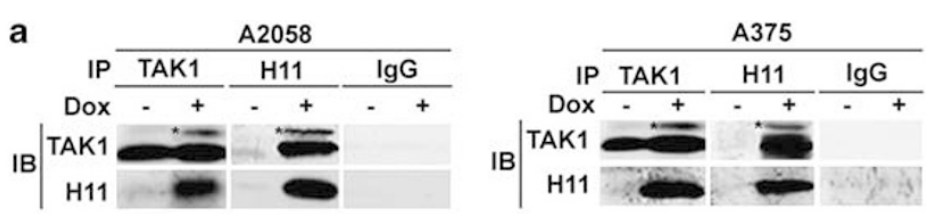

b

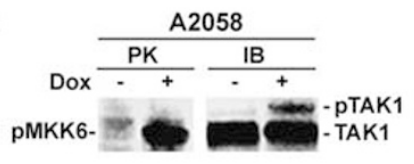

C

MeWo (P173H)

IP TAK1 H11 IgG TAK1 H11 IgG

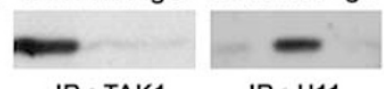

IB : TAK1

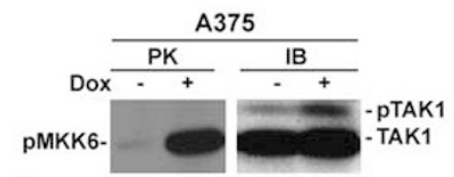

TAG51 (W51C)

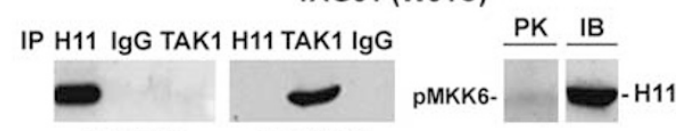

IB : H11

IB : TAK1

Figure 2 TAK1 is activated by wild-type H11/HspB8 but not its non-killing mutants. (a) Pull-down assays of untreated and Dox-treated ( $5 \mu \mathrm{g} / \mathrm{ml} ; 3$ days) stably transfected A375 and A2058 cells with H11/HspB8 or TAK1 antibodies or preimmune (Pre) IgG. (b) Immunocomplex PK assay of cell extracts as in (a) using TAK1 antibody and the TAK1 substrate MKK6 (PK). Kinase precipitates were blotted with TAK1 antibody (IB). (c) TAK1 was not activated in reciprocal pull-down assay as in (a) and immunocomplex PK assay as in (b) but using protein extracts from MeWo and TAG51 cells that respectively express the non-killing H11/HspB8 mutants, P173H and W51C

xenografts established in Balb/c (nu/nu) mice by subcutaneous (s.c.) injection of stably transfected $A 375$ and A2058 $\left(5 \times 10^{5}\right.$ or $\left.5 \times 10^{6}\right)$ cells was significantly $(P<0.001)$ inhibited in animals ( $n=10$ tumors/group) given Dox $(2 \mathrm{mg} / \mathrm{ml})$ in the drinking water as compared with those left untreated, with similar results seen for both cell lines and numbers of injected cells (Figures $1 b$ and $c$ ).

TAK1 activation is required for $\mathrm{H} 11 / \mathrm{HspB} 8$-induced melanoma cell death. Having previously shown that TAK1 is involved in $\mathrm{H} 11 / \mathrm{HspB} 8$-induced caspase-3 activation, ${ }^{9}$ we wanted to know whether its activation is required for the death of genetically diverse melanoma cells. Protein extracts from stably transfected A375 and A2058 cells treated or not with Dox ( $5 \mu \mathrm{g} / \mathrm{ml}$; 3 days) were used in reciprocal pull-down assays with TAK1 and $\mathrm{H} 11 / \mathrm{HspB} 8$ antibodies. Extracts from MeWo and TAG51 cells that constitutively express the nonkilling $\mathrm{H} 11 / \mathrm{HspB} 8$ mutants $\mathrm{P} 173 \mathrm{H}$ and $\mathrm{W} 51 \mathrm{C}^{7,9}$ served as control. H11/HspB8 co-precipitated with TAK1 from Doxtreated but not untreated A375 and A2058 cells and the precipitates contained similar levels of a slowly migrating band, which is consistent with activated TAK1 (pTAK1) (Figure 2a). Immunocomplex PK assays with the TAK1 substrate MKK6 confirmed that $\mathrm{H} 11 / \mathrm{HspB} 8$ activates TAK1 in both $A 375$ and $A 2058$ cells (Figure $2 b$ ). Dox treatment did not alter TAK1 binding to TAB $1^{9}$ or TAB2 (Supplementary Figure S3), but it inhibited the binding of TAB2 to the ubiquitin ligase tumor necrosis factor (TNF)-receptor-associated factor 6 (TRAF6) (Supplementary Figure S3), which functions in TAK1 activation by polyubiquitination. ${ }^{14} \mathrm{P} 173$ and W51C did not bind nor phosphorylate/activate TAK1 (Figure 2c), indicating that TAK1 activation is required for $\mathrm{H} 11 / \mathrm{HspB8}$-induced cell death.

A375 and A2058 cells have different levels of H11/ HspB8-induced apoptosis and caspase activation patterns. Two series of experiments were done in order to examine the role of apoptosis in $\mathrm{H} 11 / \mathrm{HspB} 8$-induced inhibition of tumor growth. First, serial sections of A2058 and A375 xenografts from Dox-treated and untreated animals as in Figures $1 b$ and $c$ were similarly stained in triple immunofluorescence. The percentage of $\mathrm{H} 11 / \mathrm{HspB} 8+$ and TUNEL + cells was calculated relative to the total number of melanoma cells $(\mathrm{S} 100+)$ and results are expressed as \% positive cells \pm S.D. $\mathrm{H} 11 / \mathrm{HspB} 8$ was expressed in virtually all the $\mathrm{S} 100+$ cells from the Doxtreated A2058 and A375 xenografts, but TUNEL co-localization was only seen in $32 \pm 10.9 \%$ of the cells from the A2058 xenografts as compared with $71.6 \pm 5.9 \%$ cells from the A375 xenografts (Figure $3 a$ ).

To examine whether the different levels of apoptosis are related to distinct patterns of caspase activation, stably transfected A375 and A2058 cells were treated or not with Dox in the presence or absence of the pancaspase inhibitor ZVAD-fmk or the caspase-3-specific inhibitor Z-DQMD-fmk and stained for TUNEL. ZVAD-fmk virtually abrogated Doxinduced apoptosis in both lines, but only one-half of this response was achieved with Z-DQMD-fmk (Figure 3b), indicating that caspase(s) other than caspase-3 contribute to $\mathrm{H} 11 / \mathrm{HspB} 8$-induced apoptosis. Immunoblotting of protein extracts from the untreated and Dox-treated xenografts (Figure 3c) and cultured cells (Figure 3d) confirmed that Dox increased the levels of activated caspase-3 (caspase-3p20/ p17) in both A375 and A2058 lines, but only A375 had activated caspase-7 (caspase-7p20). Collectively, the data indicate that the two melanoma lines have different levels of $\mathrm{H} 11 / \mathrm{HspB} 8$-induced apoptosis and suggest that caspase-7 contributes to the apoptosis of A375, but not A2058, cells.

Inflammasome-unrelated caspase-1 activation contributes to A2058 apoptosis. To determine whether a caspase other than caspase- 3 is also activated in A2058 cells, sections of the A375 and A2058 xenografts as in Figures $1 b$ and $c$ were stained in double immunofluorescence with antibodies to 

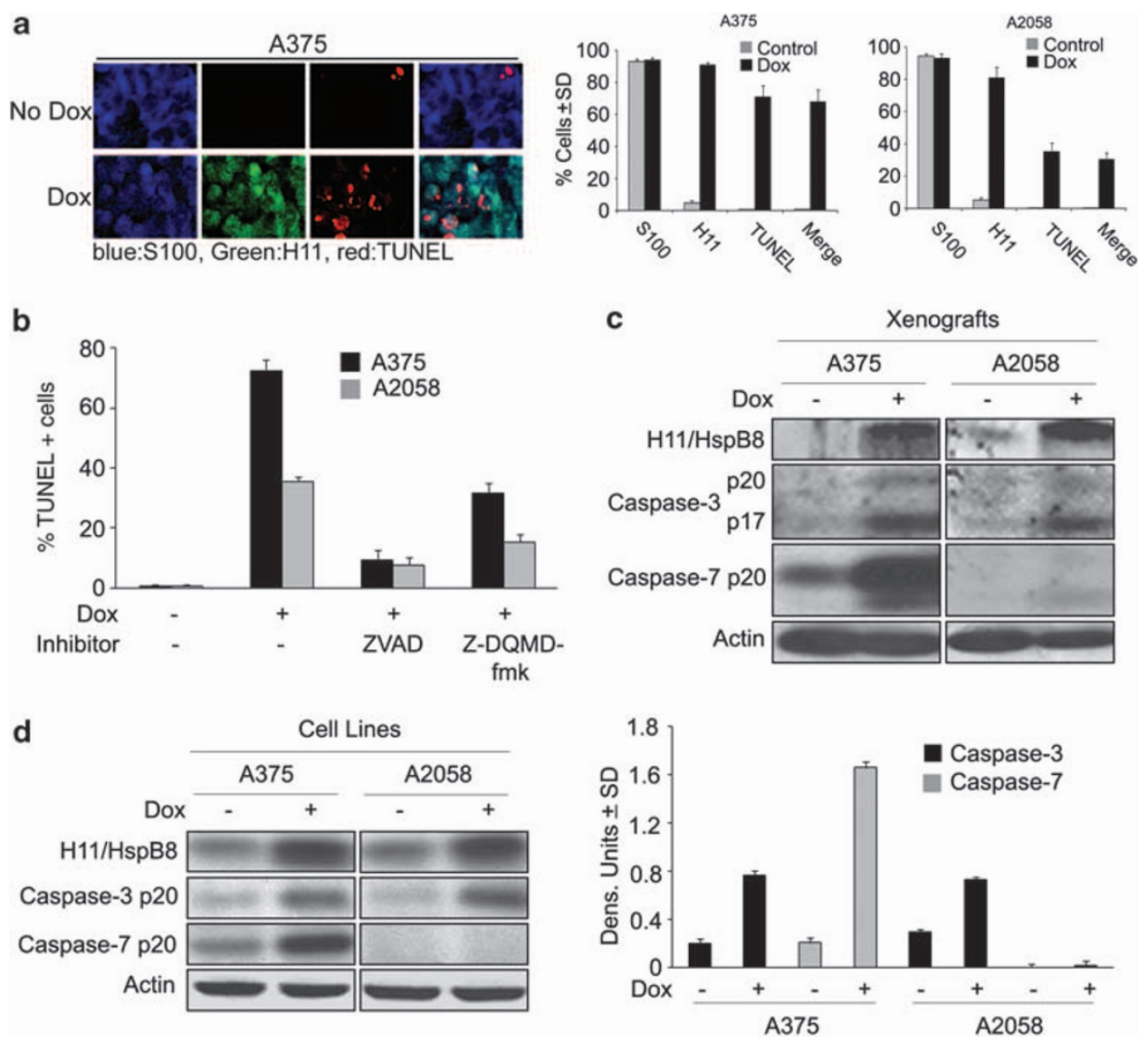

Figure 3 A375 and A2058 cells/xenografts have different apoptosis and caspase activation patterns. (a) Serial sections of xenografts collected at the end of the follow-up period from mice untreated or treated with Dox as in Figure 1b, were stained by triple immunofluorescence with antibodies to S100 (Alexafluor 350-conjugated secondary antibody; blue) and H11/HspB8 (Alexafluor 448-conjugated secondary antibody; green) and TUNEL (TMR-red labeled dUTP) and the \% positive cells calculated as in Figure 1a. (b) Stably transfected A375 and A2058 cells were treated or not with Dox ( $5 \mu \mathrm{g} / \mathrm{ml} ; 3$ days) in the presence or absence of ZVAD-fmk (20 $\mu \mathrm{M})$ or Z-DQMD-fmk $(50 \mu \mathrm{M})$ and stained by TUNEL. Data are expressed as \% TUNEL + cells \pm S.D. (c) Protein extracts from the untreated and Dox-treated xenografts as in (a) were immunoblotted with antibodies to $\mathrm{H} 11 / \mathrm{HspB} 8$, activated caspase-3 (caspase-3p20/p17) activated caspase-7 (caspase-7p20) and Actin (loading control). Blots were stripped between probing. (d) Protein extracts from stably transfected A375 and A2058 cells treated or not with Dox ( $5 \mu \mathrm{g} / \mathrm{ml} ; 3$ days) were immunoblotted as in (c). Data were quantified by densitometric scanning and results are expressed as caspase/actin densitometric units \pm S.D.

$\mathrm{H} 11 / \mathrm{HspB} 8$ and activated caspase-1 (caspase-1p20) and the percentage of cells with co-localized staining was calculated as described in Materials and Methods. Caspase-1 was studied, because it is known to cause a type of programmed cell death known as pyroptosis, which is associated with inflammasome formation and production of the inflammatory cytokines IL-1 $\beta$ and IL-18. ${ }^{15}$ Dox caused a significant increase in the percentage of cells with co-localized staining in A2058 $(1.8 \pm 0.5 \%$ and $80.1 \pm 1.9 \%$ in untreated and Dox-treated, respectively), but not $\mathrm{A} 375$ (2.5 $\pm 1 \%$ and $6.8 \pm 3.2 \%$ in untreated and Dox-treated, respectively) xenografts (Figure 4a). Immunoblotting of protein extracts from Dox-treated stably transfected cell cultures confirmed that caspase-1 was only activated in A2058 cells (Figure 4b). We conclude that activated caspase- 1 functions in apoptosis, but not pyroptosis, because (i) the percentage of TUNEL + cells was significantly decreased $(18 \pm 3.6 \%$ reduction) by the caspase-1-specific inhibitor YVAD-CHO (Figures $4 \mathrm{~b}$ and $\mathrm{c}$ ) and (ii) the cultures/xenografts were free of IL-1 $\beta$ or IL-18 although they expressed the respective pro-cytokines (shown in Figure 4d for IL-1 $\beta$ ). Cytokine production was also not seen by ELISA (sensitivity $<1 \mathrm{pg} / \mathrm{ml}$ ), confirming that caspase- 1 activation is inflammasome-independent.

H11/HspB8-induced caspase activation is TAK1-dependent. To examine whether activated TAK1 contributes to the distinct patterns of caspase activation in A375 and A2058 cells, the cultures were given the dominant-negative TAK1 mutant K63W or an empty vector control, treated with Dox ( $5 \mu \mathrm{g} / \mathrm{ml}$; 3 days) and examined for activation of caspase-3 and -7 (A375 cells) or caspase-3 and -1 (A2058 cells) by immunoblotting and immunofluorescence with antibodies to the activated forms. Dox caused a significant increase in the levels of activated caspase- 3 and -7 in A375 cells (Figure 5a) and activated caspase- 3 and -1 in A2058 cells, (Figures 5b and $d$ ), but this increase was not seen in cells given Dox together with K63W. The empty vector had no effect (Figures $5 \mathrm{a}$ and $\mathrm{b}$ ). In three independent experiments, the \% K63Wmediated inhibition, calculated relative to cells given Dox together with the empty vector, was $75-90 \%$ for all the three 

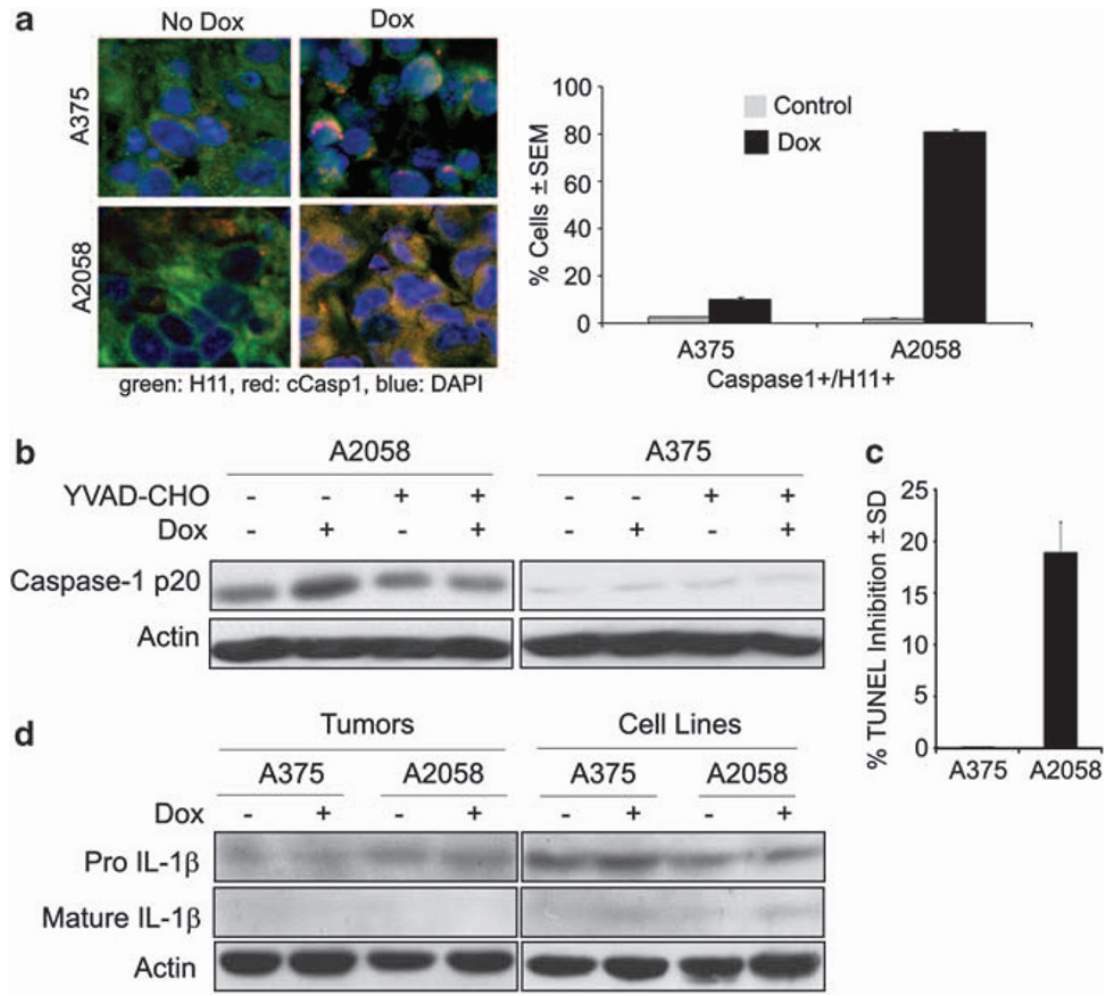

Figure 4 Inflammasome-unrelated caspase-1 activation contributes to A2058 cell apoptosis. (a) Serial sections of xenografts as in Figure $1 \mathrm{~b}$ were stained by indirect immunofluorescence with antibodies to H11/HspB8 (Alexafluor 488-conjugated secondary antibody; green) and caspase-1p20 (Casp1) (AlexaFluor 594-conjugated secondary antibody; red). Double-positive (yellow) cells were counted and the \% was calculated relative to the total cell number (determined by DAPI staining, blue). Results are expressed as \% positive cells \pm S.E.M. (b) Protein extracts from stably transfected A2058 and A375 cells untreated or treated with Dox ( $5 \mu \mathrm{g} / \mathrm{ml} ; 3$ days) in the presence or absence of the caspase-1-specific inhibitor YVAD-CHO $(20 \mu \mathrm{M})$ were immunoblotted with antibody to caspase-1p20. Blots were stripped and re-probed with actin antibody. (c) Duplicates of the YVAD-CHO-treated cultures in (b) were assayed for apoptosis by TUNEL. The cells were counted in five randomly selected $3 \mathrm{~mm}^{2}$ fields ( 250 cells each) and the $\%$ positive cells calculated relative to the total cell number. Data are expressed as $\%$ TUNEL inhibition \pm S.D. calculated from the formula [1 - YVAD-CHO-treated cells/untreated cells)] $\times 100$. (d) Extracts from A375 and A2058 xenografts as in Figure 1b (tumors) and from stably transfected A375 and A2058 cells (cell lines) treated or not with Dox ( $5 \mu \mathrm{g} / \mathrm{ml} ; 3$ days) were immunoblotted with IL-1 $\beta$ antibody, stripped and re-probed with actin antibody. Similar results were obtained for IL-18 antibody and by ELISA

caspases (Figure $5 \mathrm{c}$ ), indicating that their activation was TAK1-dependent. Because TAK1 was originally identified as an effector of TGF $\beta$-induced p38MAPK and JNK activation, but JNK is not activated in A2058 cells (Supplementary Figure S2), we next examined the effect of the p38MAPKspecific inhibitor SB20358 $(10 \mu \mathrm{M})$ on Dox-induced caspase activation. SB20358 inhibited the activation of caspase-3 in both lines, as shown in Figure 5d for A2058 cells, and caspase-7 in A375 cells (data not shown). It did not inhibit caspase-1 activation (A2058 cells; Figure 5d), suggesting that caspase- 1 is activated through another TAK1-dependent pathway.

Caspase-1 is activated by inflammasome-unrelated ASC functions. Having seen that caspase-1 activation is TAK1dependent and is not accompanied by the production of inflammatory cytokines, we considered the possibility that it involves inflammasome-unrelated ASC functions. ${ }^{16,17}$ Extracts from A2058 and A375 cells given Dox with or without the TAK1 dominant-negative mutant K63W or the empty vector control were immunoblotted with ASC antibody. The levels of ASC were significantly higher in Dox-treated than in untreated A2058 cells, an increase that was inhibited by $\mathrm{K} 63 \mathrm{~W}$, but not the empty vector. ASC or isoforms were not expressed in A375 cells (Figure 5e). Consistent with proximity-driven cleavage, ASC bound the pro- and activated forms of caspase-1 in pull-down assays, and binding was increased in Dox-treated cells (Figure 5f). ASC upregulation and caspase-1 activation were inhibited in cells given Dox together with ASC aODN $(30 \mu \mathrm{M})$, but not sODN (Figure $5 \mathrm{~g}$ ), indicating that caspase-1 activation is ASC-dependent, apparently through inflammasome-unrelated functions.

Beclin-1 is upregulated in A2058 xenografts and it contributes to apoptosis. Beclin-1 is a potent tumor suppressor that is downregulated in some human tumors and is critical in regulating autophagy and its interaction with apoptosis. ${ }^{18,19}$ Having seen that $\mathrm{H} 11 / \mathrm{HspB} 8$ inhibits the growth of $A 2058$ and $A 375$ xenografts equally well but the levels of apoptosis in A2058 tissues are relatively low, we wanted to know whether Beclin-1 is differently regulated in the two melanoma lines. Sections of A2058 and A375 xenografts as in Figures $1 \mathrm{~b}$ and $\mathrm{c}$ were stained with Beclin-1 antibody and the percentage of staining cells was calculated as described in Materials and Methods. Beclin-1 expression was minimal in both A375 and A2058 xenografts 


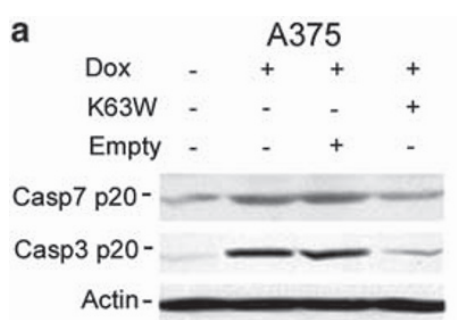

d

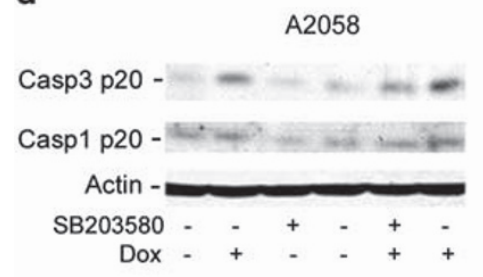

f

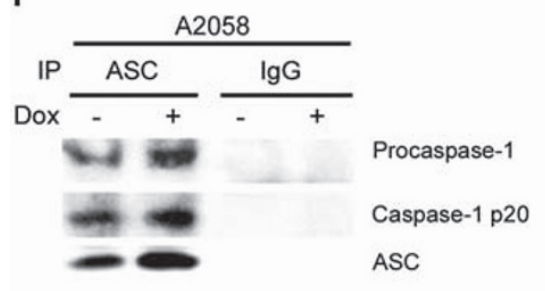

b

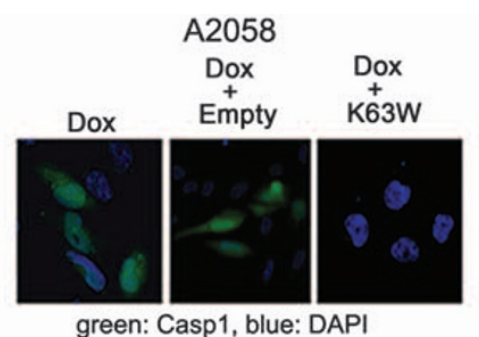

e

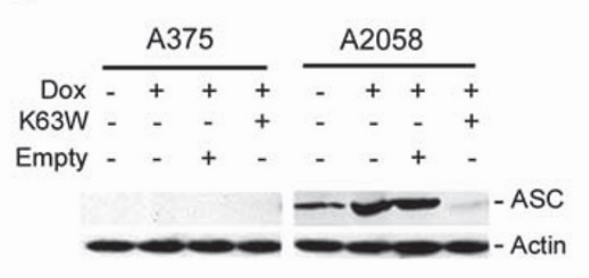

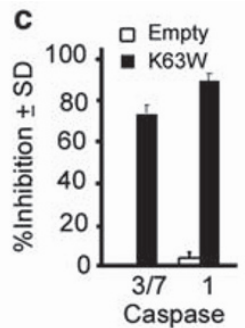

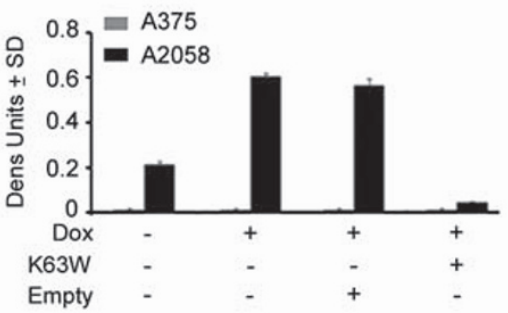

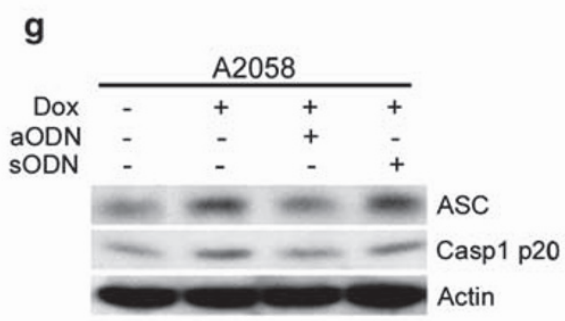

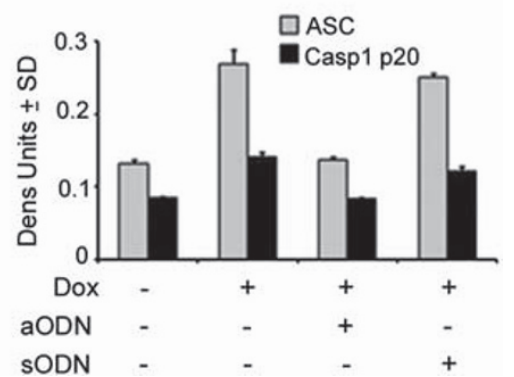

Figure 5 Caspase-1 activation is through TAK1-dependent ASC upregulation. (a) Protein extracts from stably transfected A375 cells untreated or treated with Dox $(5 \mu \mathrm{g} / \mathrm{ml} ; 3$ days) in the absence or presence of the TAK1 dominant-negative mutant K63W or the empty vector were immunoblotted with antibodies to activated caspase-7 (casp7p20), caspase-3 (casp3p20) or actin. Blots were stripped between probings. (b) Stably transfected A2058 cells as in (a) were stained with antibody to caspase-1p20 (AlexaFluor 488-conjugated secondary antibody; green) and DAPI (total cell number; blue). (c) Results for Dox-treated cells in (a) (densitometric units) and (b) (\% positive cells) were averaged for three independent experiments and the \% inhibition calculated from the formula [1 $-(\mathrm{K} 63 \mathrm{~W}$-transfected cells/untransfected cells)] $\times 100$. (d) Extracts from stably transfected A2058 cells treated or not with Dox $(5 \mu \mathrm{g} / \mathrm{ml} ; 3$ days) in the presence or absence of the p38MAPK-specific inhibitor SB203580 (10 $\mu$ M) were immunoblotted with casp3p20, antibody, sequentially stripped and re-probed with antibodies to activated caspase-1 (casp1p20) and actin. (e) Extracts from stably transfected A375 and A2058 cells treated as in (a) were immunoblotted with antibodies to ASC or actin. Data were quantified by densitometric scanning and the results are expressed as ASC/actin densitometric units \pm S.D. (f) Extracts from stably transfected A2058 cells treated or not with Dox ( $5 \mu \mathrm{g} / \mathrm{ml} ; 3$ days) were immunoprecipitated (IP) with ASC antibody or preimmune $\lg G$ and immunoblotted with antibodies to caspase 1 (recognizes the pro- and activated (p20) forms) and ASC. (g) Extracts from stably transfected A2058 cells given Dox $(5 \mu \mathrm{g} / \mathrm{ml} ; 3$ days) in the absence or presence of ASC aODN or sODN $(30 \mu \mathrm{M})$ were immunoblotted with antibodies to caspase1p20, ASC and actin. Data were quantified by densitometric scanning and the results are expressed as ASC or Casp1/actin densitometric units \pm S.D.

( $10 \pm 3 \%$ and $2 \pm 1 \%$ positive cells, respectively) but it was increased by Dox treatment, particularly in A2058 xenografts $(87.3 \pm 6.4 \%$ and $32 \pm 10.9 \%$ Beclin- $1+$ cells for A2058 and A375, respectively). A high proportion (66\%) of the TUNEL + cells $(38 \pm 6.5 \%$; Figure $3 a)$ in the A2058 xenografts were also Beclin-1 + (25.2 $\pm 1.8 \%)$, but Beclin-1/ TUNEL co-localization in A375 xenografts was minimal $(6.1 \pm 2 \%$ cells) (Figure $6 a)$, suggesting that Beclin-1 contributes to apoptosis in A2058, but not A375 tissues.

Beclin-1 upregulation is through TAK1-dependent mTOR phosphorylation at S2481. Immunoblotting of protein extracts from cells given Dox with the TAK1 dominant-negative mutant $\mathrm{K} 63 \mathrm{~W}$ or the empty vector confirmed that $\mathrm{H} 11$ / HspB8-induced Beclin-1 upregulation is TAK-1-dependent (Figure 6b). We considered the possibility that mTOR is involved in Beclin-1 upregulation because (i) the mTOR subnetwork communicates with the Beclin-1 interactome to regulate autophagy-related cancer networks that ultimately determine the life or death of cancer cells, ${ }^{19}$ (ii) TAK1 has a significant role in cancer suppression by TGF- $\beta$ signaling, ${ }^{10-12}$ and (iii) TGF- $\beta$ signaling can also activate mTOR. ${ }^{20}$ Protein extracts from A2058 and A375 cells given Dox alone or together with the TAK1 dominant-negative mutant K63W were immunoblotted with antibodies to mTOR phosphorylated at S2481 (pmTORS2481), which reflects mTORC1's intrinsic catalytic activity, or S2488 (pmTORS2448), which is the site of Akt phosphorylation and is associated with cell proliferation. ${ }^{21}$ A2058 cells had relatively low levels of pmTORS2481 that were increased by treatment with Dox, but not Dox + K63W (Figure 6c), and the empty vector had no effect (data not shown). By contrast, A375 cells had high levels of pmTORS2481 and they were not altered by Dox or Dox $+\mathrm{K} 63 \mathrm{~W}$, indicating that $\mathrm{H} 11 / \mathrm{HspB} 8$ specifically 


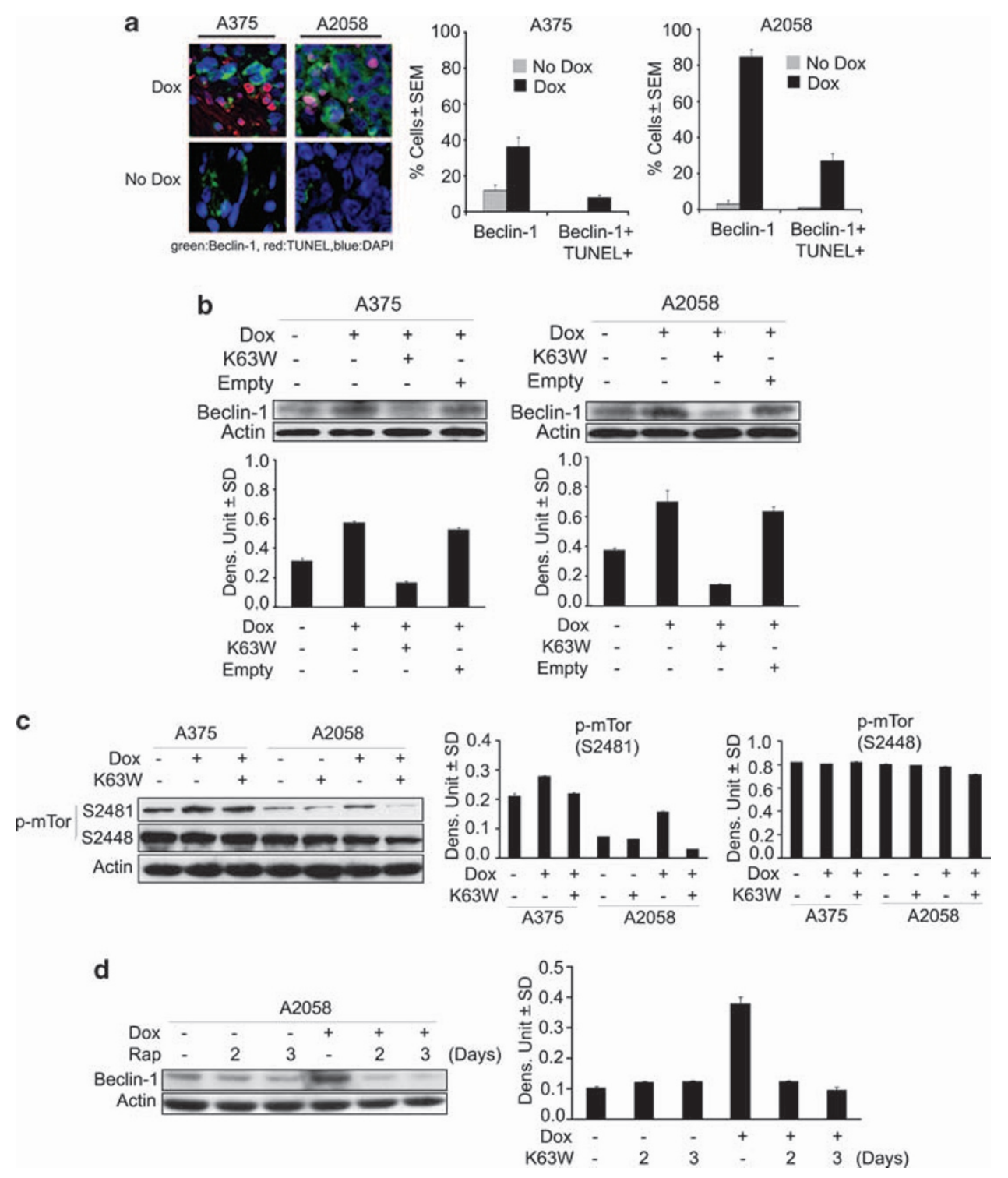

Figure 6 H11/HspB8 upregulates Beclin-1 through TAK1/pmTORS2481. (a) Serial sections from A2058 and A375 xenografts as in Figure 1b were stained by TUNEL (TMR-red labeled dUTP) and with antibody to Beclin-1 (AlexaFluor 488-conjugated secondary antibody; green) and the percentage of cells with co-localized staining was calculated relative to the total cell number determined by DAPI staining. (b) Extracts from stably transfected A375 and A2058 cells given Dox ( $5 \mu \mathrm{g} / \mathrm{ml} ; 3$ days) in the absence or presence of the TAK1 dominant-negative mutant K63W or empty vector were immunoblotted with antibodies to Beclin-1 or actin. Data were quantified by densitometric scanning and the results are expressed as Beclin-1/actin densitometric units \pm S.D. (c) Extracts from cell cultures that were untreated or Dox treated in the presence or absence of K63W were immunoblotted with antibodies to pmTORS2481, pmTORS2448 or actin. Blots were stripped between probings. Data were quantified by densitometric scanning and the results are expressed as $\mathrm{p}-\mathrm{mTOR} / \mathrm{actin}$ densitometric units \pm S.D. (d) Extracts from stably transfected A2058 cells treated or not with Dox (5 $\mu \mathrm{g} / \mathrm{ml})$ in the presence or absence of rapamycin $(20 \mathrm{nM})$ were immunoblotted with Beclin-1 followed by actin antibodies. Data were quantified by densitometric scanning and the results are expressed as Beclin-1/actin densitometric units \pm S.D.

increases pmTORS2481 in A2058 cells through TAK1 activation. This is a specific response, because the levels of pmTORS2488 were high in both lines, and they were not altered by Dox treatment (Figure 6c). Significantly, Beclin-1 upregulation was inhibited by the mTORC1 inhibitor rapamycin (20 nM) (Figure 6d), indicating that $\mathrm{H} 11 / \mathrm{HspB} 8$ upregulates Beclin-1 in A2058 cells through a TAK1/ mTORC1 pathway. The mechanism responsible for the low levels of Dox-induced Beclin-1 upregulation in A375 cells is still unclear.
Caspase-1 contributes to A2058 cell apoptosis through Beclin-1 cleavage. The Beclin-1 network regulates autophagy and apoptosis through cross-regulation that includes cleavage of Beclin- 1 by caspases- 8 or -3 and mitochondrial translocation of the cleaved fragment, sensitizing the cells to apoptosis. ${ }^{18}$ Having seen that $\mathrm{H} 11 / \mathrm{HspB} 8$ activates caspase-1 and upregulates Beclin-1 in A2058 cells, we wanted to know whether the caspase-1-mediated apoptosis seen in these cells (Figure 4b) is through Beclin-1 cleavage. In a first series of experiments, protein extracts from A375 and A2058 
a
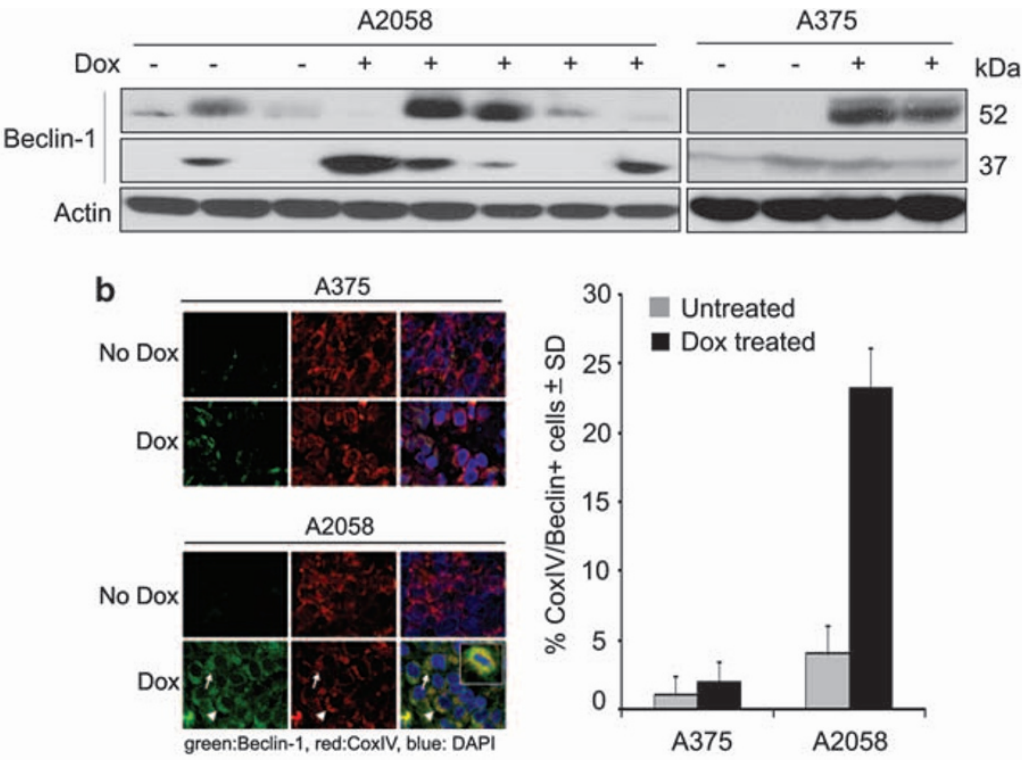

c
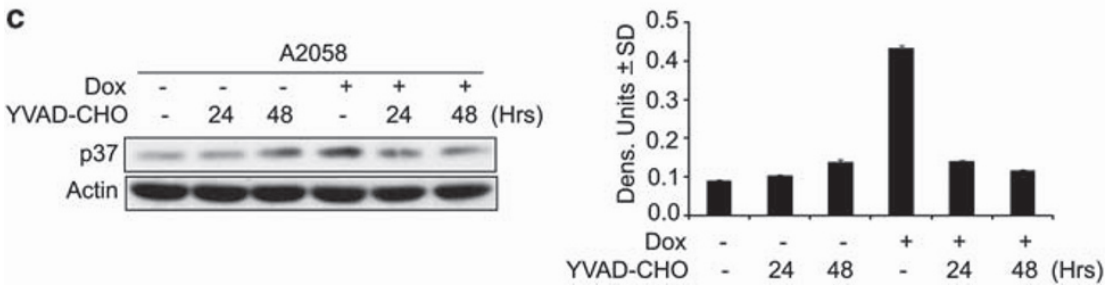

Figure 7 Caspase-1 cleaves Beclin-1 and induces apoptosis in A2058 cells. (a) Protein extracts from A375 and A2058 xenografts as in Figure $1 \mathrm{~b}$ were immunoblotted with antibodies to Beclin-1, followed by actin. Molecular weights are shown on the right. (b) Serial sections of A2058 and A375 xenografts as in Figure $1 \mathrm{~b}$ were stained with antibodies to Beclin-1 (AlexaFluor 488-conjugated secondary antibody; green) and CoxIV (mitochondrial marker; AlexaFluor 594-conjugated secondary antibody; red). The cells with CoxIV/Beclin-1 co-localization (yellow) were counted and the \% calculated relative to the total cell number (determined by DAPI staining, blue). (c) Extracts from stably transfected A2058 cells treated or not with Dox $(5 \mu \mathrm{g} / \mathrm{ml})$ in the presence or absence of the caspase-1 inhibitor YVAD-CHO $(20 \mu \mathrm{M})$, were immunoblotted with antibodies to Beclin-1 or actin and the data quantified and expressed as in Figure 6

xenografts were immunoblotted with Beclin-1 antibody and examined for the presence of the Beclin-1 p37 cleavage product. A2058 xenografts from most Dox-treated, but not untreated, animals had high levels of p37 and A375 xenografts were negative (Figure 7a). Double immunofluorescence with antibodies to Beclin-1 and the mitochondrial protein CoxIV confirmed that Beclin-1 co-localized with CoxIV in $23.3 \pm 3.4 \%$ of the cells from the Dox-treated A2058 xenografts (Figure 7b), a proportion of cells similar to that showing Beclin-1/TUNEL co-localization (25.2 $\pm 1.8 \%$; Figure 6a). Dox treatment caused a significant increase in the levels of p37 also in A2058 cultures, and the caspase-1 specific inhibitor YVAD-CHO $(20 \mu \mathrm{M})$ blocked both this increase (Figure 7c) and apoptosis (Figure 4c). The data indicate that caspase-1 cleaves Beclin-1, contributing to apoptosis in A2058 cells/tumors, but the function of Beclin-1 in the TUNEL - cells in A2058 xenografts (Figure 6a) is still unknown.

\section{Discussion}

The salient feature of the data presented in this report is the finding that restored expression of $\mathrm{H} 11 / \mathrm{HspB}$, which is silenced in a high proportion of human melanoma tissues, ${ }^{6}$ activates previously unrecognized TAK1-dependent death pathways that override melanoma genetic diversity and inhibit tumor growth. The following comments seem pertinent with respect to these findings.

Therapeutic strategies that override melanoma genetic diversity are highly desirable, but the identification of the relevant gene targets is a clinical challenge. Protein networkguided studies and global genome analyses identified genes affected by somatic copy number, amplification/overexpression, deletion/silencing or hypermethylation, but most were not reproducibly identified and their contribution to tumor growth is poorly understood.,22 Our studies follow on previous findings that the atypical $\mathrm{Hsp} \mathrm{H} 11 / \mathrm{HspB} 8$ causes cell cycle arrest in melanocytes and is consequently silenced by aberrant DNA methylation in a majority of human melanomas. ${ }^{3-7}$ We show that its restored expression overrides genetic diversity by inducing distinct death pathways, which are initiated with TAK1 activation. The site(s) of the $\mathrm{H} 11 / \mathrm{HspB} 8-$ mediated TAK1 phosphorylation and the exact contribution of the TAB proteins are still unclear. Although its ablation did not affect signaling, TAB1 binding is believed to regulate the TAK1 catalytic activity. ${ }^{23,24} \mathrm{TAB} 2$ binding recruits 

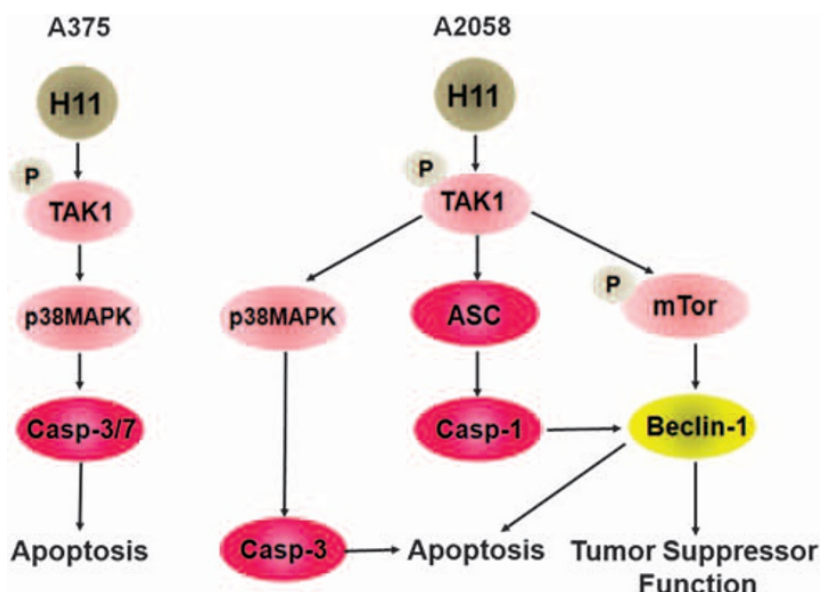

Figure 8 Schematic representation of the $\mathrm{H} 11 / \mathrm{HspB} 8$-induced death pathways in A2058 and A375 cells. H11/HspB8 activates TAK1 in both melanoma lines. In A375 cells, the TAK $1 /$ p38MAPK pathway activates caspase- $3 / 7$ to cause apoptosis. In A2058 cells, the TAK1/p38MAPK pathway activates caspase-3, but TAK1 also activates caspase-1 through ASC upregulation and upregulates Beclin-1 through mTOR phosphorylation at S2481 (pmTORS2481). Caspase-1 cleaves Beclin-1 to promote apoptosis, but Beclin-1 also contributes to cell death through still unknown tumor-suppressor functions

the ubiquitin ligase TRAF6 to TAK1, causing its activation by polyubiquitination. ${ }^{12} \mathrm{H} 11 / \mathrm{HspB} 8$ did not alter the binding of TAK1 to $\mathrm{TAB}^{9}{ }^{9}$ or TAB2, but it interfered with TAB2/TRAF6 binding (Supplementary Figure S3), thereby presumably blocking TAK1 activation by polyubiquitination. However, further studies are needed to better elucidate the mechanism of $\mathrm{H} 11 / \mathrm{HspB} 8-m e d i a t e d \mathrm{TAK} 1$ activation and determine whether it occurs only in melanoma cells.

The involvement of TAK1 in melanoma cell death is underscored by the finding that it was not activated in cells that express the $\mathrm{H} 11 / \mathrm{HspB} 8$ mutants $\mathrm{P} 173 \mathrm{H}$ and $\mathrm{W} 51 \mathrm{C}$, which do not cause cell death. ${ }^{7,9}$ Significantly, TAK1 activation was recently implicated in cancer suppression, ${ }^{10-12}$ but the downstream pathways are distinct, also when comparing A375 and A2058 cells. As schematically represented in Figure 8, apoptosis is a major component of cell death in A375 cultures/xenografts, where it is associated with the activation of TAK1/p38MAPK/caspase-3/7. Although final conclusions are precluded by the absence of a caspase-7specific inhibitor, it seems reasonable to suggest that caspase-7 contributes to the apoptosis of A375 cells, because (i) caspase- 3 and -7 are activated differently, ${ }^{25}$ (ii) maximal levels of apoptosis occur when both are activated, ${ }^{26}$ and (iii) the percentage of TUNEL + cells was significantly lower in A2058 cells, in which caspase-7 was not activated.

Recent findings suggest that the inflammasome component $\mathrm{ASC}^{15}$ can also function outside of the realm of the inflammasome, ${ }^{16,17,27}$ but the extent and spectrum of such activity are still unknown. H11/HspB8 induced TAK1-dependent ASC upregulation in A2058 cells, and it activated caspase-1, but IL-1 $\beta$ and IL-18, which are the hallmarks of the inflammasome-mediated death pathway (pyroptosis), ${ }^{15}$ were not produced. This is not an artifact related to the absence of the precursor proteins, because these were expressed in both melanoma lines, and $\mathrm{H} 11 / \mathrm{HspB} 8$ did not upregulate ASC in A375 cells, which are constitutively negative for ASC or related isoforms (Supplementary Figure 1c). The ability of signals other than $\mathrm{H} 11 / \mathrm{HspB} 8$ to stimulate the TAK1/ASC pathway is still unclear, and we do not know whether ASC upregulation involves the formation of multi-protein complexes potentially related to $\mathrm{H} 11 / \mathrm{HspB} 8$ chaperone activity. However, our data provide the first evidence in support of the recent interpretation that caspase-1 can activate substrates that are not processed during inflammation, ${ }^{28}$ and they identify the haploinsufficient tumor suppressor Beclin-1 as a caspase-1 substrate.

$\mathrm{H} 11 / \mathrm{HspB} 8$ upregulated Beclin-1 expression primarily in A2058 xenografts, where it co-localized with a high proportion $(66 \%)$ of the TUNEL + cells. Upregulation was through a TAK $1 /$ mTORC1 pathway that involved mTOR phosphorylation at S2481, which is the site of intrinsic mTORC1-specific catalytic activity. ${ }^{21}$ This effect is specific, as evidenced by the failure of $\mathrm{H} 11 / \mathrm{HspB} 8$ to increase pmTORS2488, which is the site of phosphorylation by Akt and is associated with worse prognosis and the presence of B-Raf mutations. ${ }^{29}$ Also, Dox did not alter the levels of pmTORS2481 or pmTORS2488 in untransfected A2058 or A375 cells, the latter consistent with its failure to activate Akt (Supplementary Figure S1). We conclude that Beclin-1 is an inflammation-unrelated caspase1 substrate, because (i) it was cleaved to a $37-\mathrm{kDa}$ fragment that translocated to the mitochondria and was seen in most A2058 xenografts, and (ii) the caspase-1-specific inhibitor YVAD-CHO inhibited both Beclin-1 cleavage and apoptosis. Detection of p37 in one untreated xenograft likely reflects spontaneous apoptosis. ${ }^{30}$ The failure to detect p37 in some of the Dox-treated xenografts may be due to different levels or kinetics of Beclin-1 upregulation and/or the differential contribution of Beclin-1 to apoptosis as opposed to tumor suppression. Indeed, the pronounced upregulation of Beclin-1 in A2058 xenografts, which have lower levels of $\mathrm{H} 11 / \mathrm{HspB} 8-$ induced apoptosis than their A375 counterparts, suggests that Beclin-1 contributes to tumor-growth inhibition through tumor-suppressor functions. These need further elucidation, but could include proliferative senescence, regulation of autophagic cell death networks or cell-type-specific nonapoptotic cell death induced by deregulated Ras activation. $^{18,19,31,32}$

In conclusion, we show that restored expression of $\mathrm{H} 11$ / HspB8 inhibits the growth of genetically diverse melanoma cells through the activation of distinct TAK1-dependent death pathways that include caspase- 1 activation outside of the realm of the inflammasome, mTORC1-dependent Beclin-1 upregulation and its cleavage by the activated caspase-1. In addition to supporting the interpretation that the Beclin-1 interactome interconnects with mTOR subnetworks that define the fate of cancer cells, ${ }^{19}$ our data identify TAK1 as a central player in such interconnections. Ongoing studies are designed to identify the site of TAK1 phosphorylation by $\mathrm{H} 11 / \mathrm{HspB} 8$ and verify the therapeutic potential of TAK1 in melanoma, independent of $\mathrm{H} 11 / \mathrm{HspB} 8$. Notwithstanding, the finding that H11/HspB8 activates melanoma line-specific death pathways that allow it to override genetic diversity underscores its promise as a target for epigenetic therapies. 


\section{Materials and Methods}

Cells and vectors. Melanoma cells A375 and A2058 were obtained from the American Type Culture Collection (Manassas, VA, USA) and grown in Dulbecco's modified Eagle's medium (DMEM) with 10\% fetal bovine serum (FBS, Gemini Bioproducts, Calabasas, CA, USA), $4.5 \mathrm{~g} / \mathrm{l}$ glucose, $1500 \mathrm{mg} / \mathrm{ml}$ sodium bicarbonate and $4 \mathrm{mM}$ glutamine. The cells are, respectively, homozygous and heterozygous for the constitutively active oncogenic B-RAF ${ }^{\mathrm{V} 600 \mathrm{E}}$ mutation $^{33,34}$ and they differ in the levels of the Ras-associated protein Rap $1,{ }^{35}$ activated Akt, ERK and JNK, and the expression of the JNK scaffold protein JIP-1 and ASC. ERK1/2 is activated in A375, but not A2058 cells (Supplementary Figure S1A), which also have constitutively and $\mathrm{H} 11 / \mathrm{HspB}$-activated JNK (Supplementary Figure S2), likely related to JIP1 expression (Supplementary Figure S1B). ASC is expressed in A2058, but not A375 cells (Supplementary Figure S1C). The generation and culture of A375 and A2058 cells stably transfected with tet-inducible $\mathrm{H} 11 / \mathrm{HspB} 8$ were as previously described ${ }^{9}$ and are presented in further detail in Supplementary Information Materials and Methods. MeWo cells were obtained from the American Type Culture Collection and grown in DMEM with 10\% FBS (Gemini Bioproducts) and supplemented with $4.5 \mathrm{~g} / \mathrm{l}$ glucose, $1500 \mathrm{mg} / \mathrm{ml}$ sodium bicarbonate and $4 \mathrm{mM}$ glutamine. They express the $\mathrm{H} 11 / \mathrm{HspB} 8$ mutant $\mathrm{P} 173 \mathrm{H}$ that is not methylated and does not cause melanoma cell death. ${ }^{6}$ The establishment of human embryonic kidney cells (HEK293) that stably express the H11/HspB8 mutant H11-W51C (TAG51) that also does not cause cell death was previously described. ${ }^{7}$ They were cultured in Eagle's modified minimal essential medium with $10 \%$ FBS and $400 \mu \mathrm{g} / \mathrm{ml} \mathrm{G} 418$. The TAK1 dominantnegative mutant K63W is the gift of Dr. Chen Wang (University of Texas Southwestern Medical Center, Dallas, TX, USA). K63W has a point mutation in the ATP-binding site and is kinase negative. ${ }^{35}$ The empty expression vector p3xFLAGCMV-10 was purchased from Sigma Chemicals (St. Louis, MO, USA).

Antibodies and reagents. The generation and specificity of the $\mathrm{H} 11 / \mathrm{HspB} 8$ rabbit polyclonal antibody (recognizes amino acids 181-194) were previously described. ${ }^{3,4}$ The following antibodies were purchased and used according to the manufacturer's instructions. Antibodies to Beclin-1, S100, ASC, actin, IL-1 $\beta$, IL-18 and GAPDH were from Santa Cruz Biotechnology (Santa Cruz, CA, USA). Antibodies to activated caspase-7 (caspase-7p20), caspase-3 (caspase-3p20), caspase-1 (caspase-1p20), both pro-caspase-1 and its p20 subunit, pmTOR S2481, pmTOR S2448, TAK1 and CoxIV (small subunit of mitochondrial cytochrome c oxidase) were from Cell Signaling Technology (Danvers, MA, USA). Secondary antibodies AlexaFluor488-labeled anti-mouse, AlexaFlour350-labeled anti-rabbit and AlexaFlour594-labeled anti-goat were from Invitrogen (Carlsbad, CA, USA). Doxycycline (Dox) was purchased from Clontech (Mountain View, CA, USA), and the mTORC1 inhibitor rapamycin from Cell Signaling Technology. The inhibitors specific for caspase-1 (YVAD-CHO, cell permeable), p38MAPK (SB203580) and caspase-3 (Z-DQMD-fmk) were from Calbiochem (La Jolla, CA, USA) and the pancaspase inhibitor zVAD-fmk from Promega (Madison, WI, USA). ELISA kits for IL-1 $\beta$ (R\&D Systems, Minneapolis, MN, USA) and IL-18 (Invitrogen; sensitivity $>1 \mathrm{pg} / \mathrm{ml}$ ) were used as per the manufacturer's instructions.

Animal studies. The Animal Care and Use committee of the University of Maryland School of Medicine approved all the described studies. Six-eightweek-old female nude mice (Balb/c nu/nu) were obtained from Charles River Laboratories (Wilmington, MA, USA). To establish melanoma xenografts, cells stably transfected with tet-inducible $\mathrm{H} 11 / \mathrm{HspB} 8\left(5 \times 10^{5}\right.$ or $5 \times 10^{6}$ in $\left.100 \mu \mathrm{l}\right)$ were injected subcutaneously into both hind flanks. Animals were randomly assigned to two distinct groups ( $n=10$ tumors each), one of which was given $2 \mathrm{mg} / \mathrm{ml}$ Dox in the drinking water containing $5 \%$ sucrose, while the other was left untreated. Minimum and maximum perpendicular tumor axes were measured with a microcalipers weekly (for $5 \times 10^{5}$ cells) or every $2-4$ days (for $5 \times 10^{6}$ cells) and tumor volume was calculated using the formula: volume $=\left[\left(\right.\right.$ length $\times$ width $\left.\left.^{2}\right) / 2\right]$. Animals were maintained in pathogen-free conditions and euthanized when their tumors reached $1.5 \mathrm{~cm}$ in any one direction. Tissues collected after euthanasia were processed for staining and immunoblotting.

TUNEL assay. The In situ Cell Death Detection Kit (TUNEL) with TMR-red labeled dUTP was purchased from Roche (Indianapolis, IN, USA) and used according to the manufacturers' instructions. Stained cells were counted in five randomly selected $3 \mathrm{~mm}^{2}$ fields (250 cells each), and the \% positive cells calculated relative to the total cell number.
Immunofluorescence, immunoprecipitation and immunoblotting. Immunofluorescent staining was as previously described. ${ }^{9}$ Stained cells were counted in five randomly selected $3 \mathrm{~mm}^{2}$ fields ( 250 cells each) and the \% positive cells calculated relative to the total number of cells. Protein extraction and immunoblotting or immunoprecipitation/immunoblotting (pull-down) assays were done as previously described. ${ }^{3,4,9}$ Further details are provided in Supplementary Materials and Methods.

Immunocomplex protein kinase (PK) assays. Immunocomplex PK assays were as described ${ }^{3,9}$ using MKK6 (1 $1 \mu \mathrm{g}$; Upstate Biotechnology, Lake Placid, NY, USA) as the TAK1 kinase substrate and $10 \mu \mathrm{Ci}\left[{ }^{32} \mathrm{P}\right]-\mathrm{ATP}$ ( $3000 \mathrm{C} / \mathrm{mM}$; Perkin Elmer, Waltham, MA, USA).

Antisense oligonucleotides. Phosphorothioate oligodeoxynucleotides (ODNs) complementary to the ASC translation initiation site (antisense ODN (aODN); $5^{\prime}$-CGC CCCATGGCT- $3^{\prime}$ ) or sense ODN (sODN; $5^{\prime}$-AGCCATGGGGCG-3') were obtained from Integrated DNA Technologies (Coralville, IA, USA). Cells in triplicate were exposed to Dox ( $5 \mu \mathrm{g} / \mathrm{ml} ; 3$ days) in the presence or absence of the ODNs $(30 \mu \mathrm{M})$ in a medium with $10 \%$ heat-inactivated FBS $\left(30 \mathrm{~min}\right.$ at $65^{\circ} \mathrm{C}$ to destroy nuclease activity). ${ }^{7}$

Statistical analysis. Analysis of variance (ANOVA) was performed with SigmaStat version 3.1 for Windows (Systat Software, Point Richmond, CA, USA). Tumor volumes were compared over time between untreated and treated groups by pairwise two-way ANOVA followed by the Tukey's honestly significant difference test.

\section{Conflict of Interest}

The authors declare no conflict of interest.

Acknowledgements. These studies were supported by Public Health Service grant AR053512 from NIAMS, National Institutes of Health (NIH).

1. Erdei E, Torres SM. A new understanding in the epidemiology of melanoma. Exp Rev Anticancer Ther 2010; 10: 1811-1823.

2. Sigalotti L, Covre A, Fratta E, Paris G, Colizzi F, Rizzo A et al. Epigenetics for human cutaneous melanoma: setting the stage for new therapeutic strategies. $J$ Trans/ Med 2010; 8: 56.

3. Smith CC, Yu YX, Kulka M, Aurelian L. A novel human gene similar to the protein kinase (PK) coding domain of the large subunit of herpes simplex virus type 2 ribonucleotide reductase (ICP10) codes for a serine-threonine PK and is expressed in melanoma cells. J Biol Chem 2000; 275: 25690-25699.

4. Aurelian L, Smith CC, Winchurch R, Kulka M, Gyotoku T, Zaccaro L et al. A novel gene expressed in human keratinocytes with long-term in vitro growth potential is required for cell growth. J Invest Dermatol 2001; 116: 286-295.

5. Sui X, Li D, Qiu H, Gaussin V, Depre C. Activation of the bone morphogenetic protein receptor by $\mathrm{H} 11$ kinase/Hsp22 promotes cardiac cell growth and survival. Circ Res 2009; 104: 887-895.

6. Smith CC, Li B, Liu J, Lee KS, Aurelian L. The levels of H11/HspB8 DNA methylation in human melanoma tissues and xenografts are a critical molecular marker for 5-Aza-2'-deoxycytidine therapy. Cancer Invest 2011; 29: 383-395.

7. Gober MD, Smith CC, Ueda K, Toretsky JA, Aurelian L. Forced expression of the $\mathrm{H} 11$ heat shock protein can be regulated by DNA methylation and trigger apoptosis in human cells. J Biol Chem 2003; 278: 37600-37609.

8. Cui XY, Wang N, Yang BX, Gao WF, Lin YM, Yao XR et al. HSPB8 is methylated in hematopoietic malignancies and overexpression of HSPB8 exhibits antileukemia effect. Exp Hematol 2012; 40: 14-21.

9. Li B, Smith CC, Laing JM, Gober MD, Liu L, Aurelian L. Overload of the heat-shock protein $\mathrm{H} 11 / \mathrm{HspB} 8$ triggers melanoma cell apoptosis through activation of transforming growth factor-beta-activated kinase 1. Oncogene 2007; 26: 3521-3531.

10. Heldin CH, Landström M, Moustakas A. Mechanism of TGF-beta signaling to growth arrest, apoptosis, and epithelial-mesenchymal transition. Curr Opin Cell Biol 2009; 21: 166-176.

11. Bettermann K, Vucur M, Haybaeck J, Koppe C, Janssen J, Heymann F et al. TAK1 suppresses a NEMO-dependent but NF-kappaB-independent pathway to liver cancer. Cancer Cell 2010; 17: 481-496.

12. Thakur N, Sorrentino A, Heldin CH, Landström M. TGF-beta uses the E3-ligase TRAF6 to turn on the kinase TAK1 to kill prostate cancer cells. Future Oncol 2009; 5: 1-3.

13. Shieh JM, Huang TF, Hung CF, Chou KH, Tsai YJ, Wu WB. Activation of C-Jun N-terminal kinase is essential for mitochondrial membrane potential change and apoptosis induced by doxycycline in melanoma cells. Br J Pharmacol 2010; 160: 1171-1184. 
14. Shim JH, Xiao C, Paschal AE, Bailey ST, Rao P, Hayden MS et al. TAK1, but not TAB1 or TAB2, plays an essential role in multiple signaling pathways in vivo. Genes Dev 2005; 19 : 2668-2681.

15. Davis BK, Wen H, Ting JP. The inflammasome NLRs in immunity, inflammation, and associated diseases. Annu Rev Immunol 2011; 29: 707-735.

16. Taxman DJ, Holley-Guthrie EA, Huang MT, Moore CB, Bergstralh DT, Allen IC et al. The NLR adaptor ASC/PYCARD regulates DUSP10, mitogen-activated protein kinase (MAPK), and chemokine induction independent of the inflammasome. $J$ Biol Chem 2011; 286: 19605-19616.

17. Ippagunta SK, Malireddi RKS, Shaw PJ, Neale GA, Walle LV, Green DR et al. The inflammasome adaptor ASC regulates the function of adaptive immune cells by controlling Dock2-mediated Rac activation and actin polymerization. Nat Immunol 2011; 12: 1010-1016.

18. Kang R, Zeh HJ, Lotze MT, Tang D. The Beclin 1 network regulates autophagy and apoptosis. Cell Death Differ 2011; 18: 571-580.

19. Wang SY, Yu QJ, Zhang RD, Liu B. Core signaling pathways of survival/death in autophagy-related cancer networks. Int J Biochem Cell Biol 2011; 43: 1263-1266.

20. Das F, Ghosh-Choudhury N, Mahimainathan L, Venkatesan B, Feliers D, Riley DJ et al. Raptor-rictor axis in TGF $\beta$-induced protein synthesis. Cell Signal 2008; 20: 409-423.

21. Soliman GA, Acosta-Jaquez HA, Dunlop EA, Ekim B, Maj NE, Tee AR et al. mTOR Ser2481 autophosphorylation monitors mTORC-specific catalytic activity and clarifies rapamycin mechanism of action. J Biol Chem 2010; 285: 7866-7879.

22. Valsesia A, Rimoldi D, Martinet D, Ibberson M, Benaglio P, Quadroni M et al. Network guided analysis of genes with altered somatic copy number and gene expression reveals pathways commonly perturbed in metastatic melanoma. PLOS One 2011; 6: e18369.

23. Inagaki M, Omori E, Kim JY, Komatsu Y, Scott G, Ray MK et al. TAK1-binding protein 1, TAB1, mediates osmotic stress-induced TAK1 activation but is dispensable for TAK1mediated cytokine signaling. J Biol Chem 2008; 283: 33080-33086.

24. Panka DJ, Sullivan RJ, Mier JW. An inexpensive, specific and highly sensitive protocol to detect the BrafV600E mutation in melanoma tumor biopsies and blood. Melanoma 2010; 20: 401-407.

25. Lamkanfi M, Kalai M, Saelens X, Declercq W, Vandenabeele P. Caspase-1 activates nuclear factor of the kappa-enhancer in B cells independently of its enzymatic activity. J Biol Chem 2004; 279: 24785-24793.

26. Sung YH, Lee JS, Park SH, Koo J, Lee GM. Influence of co-down-regulation of caspase-3 and caspase-7 by siRNAs on sodium butyrate-induced apoptotic cell death of Chinese hamster ovary cells producing thrombopoietin. Metab Eng 2007; 9: 452-464.
27. Motani K, Kushiyama H, Imamura R, Kinoshita T, Nishiuchi T, Suda T. Caspase-1 protein induces apoptosis-associated speck-like protein containing a caspase recruitment domain (ASC)-mediated necrosis independently of its catalytic activity. J Biol Chem 2011; 286 : 33963-33972.

28. Walsh JG, Logue SE, Luthi AU, Martin SJ. Caspase-1 promiscuity is counterbalanced by rapid inactivation of the processed enzyme. I Biol Chem 2011; 286: 32513-32524.

29. Pópulo H, Lopes JM, Soares $\mathrm{P}$. The mTOR signalling pathway in human cancer. Int $\mathrm{J}$ Mol Sci 2012; 13: 1886-1918.

30. Cichorek M, Kozłowska K, Wachulska M, Zielińska K. Spontaneous apoptosis of melanotic and amelanotic melanoma cells in different phases of cell cycle: relation to tumor growth. Folia Histochem Cytobiol 2006; 44: 31-36.

31. Overmeyer JH, Kaul A, Johnson EE, Maltese WA. Active ras triggers death in glioblastoma cells through hyperstimulation of macropinocytosis. Mol Cancer Res 2008; 6: 965-977.

32. Young AR, Narita M, Ferreira M, Kirschner K, Sadaie M, Darot JF et al. Autophagy mediates the mitotic senescence transition. Genes Dev 2009; 23: 798-803.

33. Maddodi N, Bhat KM, Devi S, Zhang SC, Setaluri V. Oncogenic BRAFV600E induces expression of neuronal differentiation marker MAP2 in melanoma cells by promoter demethylation and down-regulation of transcription repressor HES1. J Biol Chem 2010; 285: 242-254.

34. Gao L, Feng Y, Bowers R, Becker-Hapak M, Gardner J, Council L et al. Ras-associated protein-1 regulates extracellular signal-regulated kinase activation and migration in melanoma cells: two processes important to melanoma tumorigenesis and metastasis. Cancer Res 2006; 66: 7880-7888.

35. Diao L, Zhang B, Xuan C, Sun S, Yang K, Tang Y et al. Activation of cJun N-terminal kinase (JNK) pathway by HSV-1 immediate early protein ICPO. Exp Cell Res 2005; 308: $196-210$.

Cell Death and Disease is an open-access journal published by Nature Publishing Group. This work is licensed under the Creative Commons Attribution-NonCommercial-No Derivative Works 3.0 Unported License. To view a copy of this license, visit http://creativecommons.org/licenses/by-nc-nd/3.0/

\section{Supplementary Information accompanies the paper on Cell Death and Disease website (http://www.nature.com/cddis)}

